\title{
Führungsforschung im Bildungsbereich: Schulleitung im Spannungsfeld erweiterter Rechte und Pflichten
}

\author{
Stefan Brauckmann • Ferdinand Eder
}

Angenommen: 23. Mai 2019 / Online publiziert: 13. Juni 2019

(C) Der/die Autor(en) 2019

Zusammenfassung Auffällig ist, dass die veränderte Aufgabenstruktur von Schulleitung, die dem Konzept der Neuen Steuerung folgt, bisher nur vereinzelt aufgegriffen wurde, um Schulleitungshandeln zu analysieren und Unterstützungsbedarf von SchulleiterInnen zur Wahrnehmung von Führungsaufgaben im Rahmen erweiterter schulischer Eigenverantwortung zu identifizieren. Dementsprechend wird mit dem hier vorliegenden Beitrag beabsichtigt, die Handlungsrelevanz und den Anwendungsbezug Neuer Steuerungsansätze und -instrumente für das Schulleitungshandeln zu verdeutlichen. Es werden Beziehungs- und Zusammenhangsstrukturen zwischen Neuen Steuerungsansätzen und Schulleitungshandeln skizziert. Besondere Aufmerksamkeit gilt der Frage nach der bestmöglichen Kombinierbarkeit solcher Aufgaben und Handlungsfeldern schulischer Führung, die sich auf die Verbesserung von Schul- und Unterrichtsqualität richten. Das soll einem Verständnis von Schulleitungshandeln zugutekommen, das über die prominenten effektiven Führungsstile (unterrichtsbezogener und transformationaler Führungsstil) hinausgeht und kontextwie situationsbezogene Formen der Kombination von Führungsstilen erfasst.

Schlüsselwörter Neue Steuerung · Schulqualität · Führungshandeln · Rechenschaftspflicht $\cdot$ Autonomie $\cdot$ Educational Governance

\footnotetext{
Univ.- Prof. Dr. S. Brauckmann $(\bowtie)$

Institut für Unterrichts- und Schulentwicklung (IUS), Alpen-Adria-Universität Klagenfurt, Sterneckstraße 15, 9010 Klagenfurt, Österreich

E-Mail: Stefan.Brauckmann@aau.at

Univ. Prof. i.R. Dr. F. Eder

Fachbereich Erziehungswissenschaft, Universität Salzburg, Erzabt-Klotz-Straße 1, 5020 Salzburg, Österreich
} 


\title{
Leadership research in education: Activities of school principals between extended rights and responsibilities
}

\begin{abstract}
It is noticeable that the changed task structure of school leadership, which follows the concept of the New Public Management, has so far only been taken up sporadically in order to analyze the actions of school principals and to identify their support needs in the performance of leadership tasks within the framework of extended school self-responsibility. Accordingly, the present contribution aims to clarify the relevance and application of New Public Management Approaches and their respective instruments for school principals. Relationship and connection structures between new public management approaches and school leadership practices are outlined. Particular attention will be paid to the question of the best possible combination of such tasks and fields of action of school leadership that are aimed at improving school and teaching quality. This should benefit an understanding of school leadership that goes beyond the prominent effective leadership styles (instructional and transformational leadership style) and encompasses context- and situation-related forms of combining leadership styles.
\end{abstract}

Keywords New Public Management · School Quality · Leadership actions · Accountability $\cdot$ Autonomy $\cdot$ Educational Governance

\section{Erwartungen an Führungshandeln im Kontext Neuer Steuerungskonzepte}

In den letzten beiden Jahrzehnten wurde die Gestaltung des Schulsystems stärker in Verbindung mit der Entwicklung der öffentlichen allgemeinbildenden Einzelschule (vgl. van Ackeren und Klemm 2009; Rolff 2009) diskutiert. Zu den inhaltlichen wie analytischen Schwerpunkten zählten dabei im deutschsprachigen Forschungsraum (vgl. Brauckmann et al. 2010) Dezentralisierung, Markt-/Wettbewerbsmechanismen, Schulentwicklung, externe Evaluation und institutionelle Rechenschaftslegung. Bezogen auf die Einzelschule bedeutete das Forschungsarbeit zur institutionellen Autonomie, zu alternativen Finanzierungsformen, zum Umgang mit Evaluationsergebnissen und zur institutionellen Rechenschaftslegung. Hinzu kam das bis zur kommunalen Ebene reichende und mit systemischer Rechenschaftslegung verbundene Bildungsmonitoring (vgl. Böttcher et al. 2008; Specht 2008).

Neue Steuerung bedeutet im Kontext dieser Forschung den Versuch, Antwort(en) auf Herausforderungen zu geben, die mit herkömmlicher Steuerung nicht mehr hinreichend verarbeitet werden können. Auch die Art und Weise, wie bisher Arbeitsprozesse innerhalb der Schule strukturiert und organisiert wurden, erscheint den veränderten Systembedingungen nicht mehr angemessen (vgl. Pont et al. 2008). Kennzeichnend für das Neue Steuerungskonzept ist es, dass es den Schulen erweiterte Handlungsspielräume bietet, aber verlangt, dass die Einrichtungen offenlegen und nachweisen müssen, wie sie den eigenverantwortlichen Einsatz von Ressourcen und ihre pädagogische Eigenständigkeit ausweislich ihres Schulprogramms und des Schulprofils im Hinblick auf die Erreichung qualitätsorientierter Bezugsnormen 
nutzen. Die verschiedenen Formen von Evaluation dienen folglich der Qualitätssteigerung (Improvement) und der Rechenschaft (Accountability). Erweiterte Eigenständigkeit der Schulen und externe Kontrolle schulischer Ergebnisse bei zunehmend datengestützten, kriterialen Vergleichen von SchülerInnenleistungen bedingen einander.

In der Forschung werden beide Regelungsansätze nicht nur unter den Aspekten ihrer gegenseitigen Stützung, sondern auch hinsichtlich widersprüchlicher Wechselwirkungen diskutiert (vgl. Klein 2017; Avenarius und Füssel 2008; van Ackeren und Bellenberg 2004). Mit Blick auf bisherige Konzepte haben BeobachterInnen auf die Prätention in der Begriffswahl für das derzeit bewegende Steuerungskonzept hingewiesen. So scheint ,die gängige Auslegung des alten Steuerungsmodells“ (Herrmann 2009, S. 59) als staatlich-bürokratische Steuerung ,,vor allem am Profilierungsnutzen für das neue Modell orientiert zu sein“ (ebd. 2009, S. 59). Ebenso wird vorgebracht, dass es auch unter der alten, vermeintlich strikt hierarchisch agierenden staatlichbürokratischen Steuerungsmacht schon immer Raum für kontextspezifische Ausnahmeregelungen gab, ,die mit normierenden Einheitsverordnungen nicht zu bearbeiten waren“ (ebd. 2009, S. 65 f.).

Dennoch bedeutet das Konzept der Neuen Steuerung mit der Erweiterung von Handlungsspielräumen, der Standardsetzung und vermehrter Rechenschaftspflicht zu normativen Handlungsvorgaben eine deutliche Veränderung im Schulleitungsverständnis (vgl. Kanape und Kemethofer 2017; Altrichter und Kemethofer 2015). Diese erweist sich nicht zuletzt mit Blick auf die Anforderungsprofile für schulische Leitungsfunktionen (vgl. Brauckmann 2016; Warwas 2012). An Schulen mit mehr Eigenverantwortung stehen Schulleitungen somit vor neuen strategischen Führungsaufgaben, müssen über das operative Management hinaus auch die Ziele jeweiliger Schulentwicklung formulieren und deren konzeptionelle Umsetzung mit Blick auf die extern herangetragenen Standards betreiben (vgl. Bonsen 2016). Eine umfassende Organisationsentwicklung verlangt zudem auch die Analyse und Nutzung von Daten. Grundsätzlich werden die SchulleiterInnen als IdeengeberInnen von Entwicklungsaktivitäten adressiert, die Veränderungsprozesse in der Schule initiieren und durchsetzen können. Ihnen fällt eine multiple Rolle als VeränderungsagentInnen, WissensvermittlerInnen und VerfahrenshelferInnen zu (vgl. Wissinger 2016; Schratz et al. 2016).

In der Praxis bedeutet dies, dass sich für die Leitung an Schulen auch der Grad ihrer Steuerungsverantwortung erhöht. Die erweiterten Entscheidungsmöglichkeiten und Gestaltungsoptionen sowie die wachsenden Rechenschaftspflichten erfordern auf einzelschulischer Ebene die Übernahme neuer Management- und Führungsaufgaben. Nur so kann die Komplexität der schulischen Probleme zugunsten einer effizienten Organisationssteuerung und der Delegation von personalstrategischer Führung besser einzelschulisch bewältigt werden. Alles in allem fällt es Schulleitungen zu, die zur Umsetzung der qualitätsbezogenen Eigensteuerung von Schule übertragenen Befugnisse gestaltend zu nutzen und in der Schule konkret werden zu lassen.

Die Handlungsoptionen und damit auch die Rollen der Schulleitungen unterliegen länderspezifischen Regelungen und unterscheiden sich entsprechend. Das betrifft folgende Aufgabenbereiche: 
- Personalführung und Organisationsentwicklung (z.B. Einstellung und Entwicklung von Personal)

- Unterricht und pädagogische Innovation (z. B. Variationen in der Unterrichtsorganisation sowie -durchführung)

- Verwaltungs- und Organisationsaufgaben (z.B. schuleigene Budgetierung von Lehr- und Lernmitteln)

- Öffnung von Schule (z. B. Kooperation mit Unternehmen, Arbeit in Schulnetzwerken) (vgl. Thillmann et al. 2015)

Über ihr Tradiertes hinaus weist die neue Steuerungsphilosophie der Schulleitung weitere Aufgaben zu. Sie trägt wie bisher Verantwortung für die Erarbeitung und Umsetzung eines eigenständigen Profils und eines diesem angemessenen pädagogischen Konzepts. Um den Zielen der Qualitätssicherung und -verbesserung gerecht zu werden, erhalten Schulen und Schulleitung gegebenenfalls zusätzliche materielle Ressourcen - gemäß dem Leitbild der eigenverantwortlichen Schule sollen gröBere Befugnisse bei der schuleigenen Personalauswahl und der Verwendung von Personal- und Sachmitteln ermöglicht werden (vgl. Altrichter et al. 2016). Eine bloBe Selbststeuerung der Schulen ist damit jedoch noch nicht beabsichtigt. Vielmehr gehen erweiterte personelle, finanzielle und administrative Eigenständigkeit sowie größere pädagogische Gestaltungsoptionen der Schulen mit Handlungsanweisungen der zuständigen Schulverwaltungsbehörden an die Schulen einher. Diese Weisungen können als Restriktionen der erweiterten schulischen Autonomie verstanden werden, wobei Schulprogramme, Zielvereinbarungen oder sonstige vertragsähnliche Vereinbarungen zwischen Schulverwaltung und Schule als ,institutionelle Arrangements“ dienen (Heinrich und Kussau 2016).

In der Literatur ist verschiedentlich auf Paradoxien, Widersprüche und Dilemmata hinsichtlich des Verhältnisses von Steuerungstheorie und Steuerungspraxis hingewiesen worden. Vor allem konnte noch nicht zufriedenstellend geklärt werden, inwieweit die Annahmen zur Wirkung der Neuen Steuerungskonzepte überhaupt gerechtfertigt sind (vgl. Bellmann 2016; Schümer und Weiss 2008; de Grauwe 2004). Rhetorik und Realität Neuer Steuerung fallen zurzeit in vielerlei Hinsicht noch auseinander.

\section{Zur theoretischen Fundierung wirksamen Führungshandelns}

Neben der häufig übergreifend beschriebenen theoretischen Dimension haben die neuen Steuerungskonzepte erhebliche schulpraktische Implikationen in Bezug auf die Erfüllung übergeordneter Zielvorstellungen. In diesem Zusammenhang ist die Rolle von Schulleitung als zentrale/r AkteurIn zunehmend ins Blickfeld der empirischen Schulleitungsforschung gerückt. Ihr geht es um Aufschluss über die praktische Bewährung neuer Regelungsansätze, die auf die Steigerung und Sicherung schulischer Qualität abheben (Böttcher 2017).

In dem Maße, in dem das Wirken der Schulleitung stärker aus einer Gesamtsteuerungsperspektive betrachtet wird, gewinnt die ergebnisorientierte Wirksamkeit schulischen Führungshandelns an Bedeutung. Die Befundlage der empirischen 
Tab. 1 Übersicht: Beschreibung von Führungskonzepten (Schratz et al. 2016, S. 225)

\begin{tabular}{|c|c|}
\hline $\begin{array}{l}\text { System Leadership } \\
\text { Hopkins (2008), } \\
\text { Huber (2010), Schley } \\
\text { und Schratz (2010) }\end{array}$ & $\begin{array}{l}\text { Fokus auf Erfolg des Schulsystems einer Region bzw. mehrerer Subsys- } \\
\text { teme, Förderung von Schulgemeinschaften, Verringerung der Schere zwi- } \\
\text { schen besonders guten und schlechten Schulen, Mitwirkung an Schulent- } \\
\text { wicklungsprojekten und Modellvorhaben in anderen Schulen, Führung wird } \\
\text { funktional gesehen und nicht personenorientiert }\end{array}$ \\
\hline $\begin{array}{l}\text { Geteilte Führung, } \\
\text { Distributed Leadership } \\
\text { Gronn (2000) }\end{array}$ & $\begin{array}{l}\text { Delegation erfolgt in den Aufgaben, Kompetenzen, Entscheidungsberei- } \\
\text { chen und Verantwortungen, die Entscheidungsinhalte werden mit maßgeb- } \\
\text { licher Beteiligung bzw. in weitgehender Selbstständigkeit der Geführten } \\
\text { festgelegt, die Ausrichtung von Zielen bzw. die Zielfestlegung erfolgt durch } \\
\text { die Führung, hohes Vertrauen in die Kompetenzen der Geführten notwen- } \\
\text { dig, aufwändige Planungs- und Controllingaktivitäten }\end{array}$ \\
\hline $\begin{array}{l}\text { Instruktionale (unter- } \\
\text { richtsbezogene) Führung } \\
\text { De Bevoise (1984), } \\
\text { Smith und Andrews } \\
\text { (1989) }\end{array}$ & $\begin{array}{l}\text { Orientierung an der Unterrichtsqualität und am Lernfortschritt der Schüle- } \\
\text { rinnen und Schüler, Kommunikation klarer schulischer und pädagogischer } \\
\text { Ziele, Förderung von kooperativen Beziehungen im Kollegium, direkte } \\
\text { Einflussnahme auf den Unterricht, Führung als „Ausbildung“, hohe Trans- } \\
\text { parenz }\end{array}$ \\
\hline $\begin{array}{l}\text { Transformationale } \\
\text { Führung } \\
\text { Burns (1978), Bass } \\
\text { (1985) }\end{array}$ & $\begin{array}{l}\text { Vermittlung attraktiver Visionen und inspirierender Motivierung, Entwick- } \\
\text { lung von gemeinsamen Visionen, Herstellung von gemeinsamen Werten, } \\
\text { Förderung einer kooperativen und professionellen Lernkultur, aktive Per- } \\
\text { sonalentwicklung, Förderung von Problemlöse- und Umsetzungsstrategien, } \\
\text { Führung als „,Vorbild“, hohe Transparenz, Entwicklung einer „,lernenden } \\
\text { Organisation“, Bedeutung für die Schule als Gesamtorganisation }\end{array}$ \\
\hline
\end{tabular}

Schulleitungsforschung zeigt, dass bisher vorwiegend die Wirkungen von effektiven Führungsstilen auf den schulischen Output untersucht wurden (vgl. Pietsch und Tulowitzki 2017; Bonsen 2016; Hattie 2009). Zu ihrer theoretischen Fundierung sind mit der Betonung von Management- und Organisationswissen und dem expliziten Bezug auf Themen der Organisationsentwicklung wesentlich betriebswirtschaftliche Vorbilder adaptiert worden (vgl. Heinrich und Kohlstock 2016). Führungskonzeptionen, wie sie vor diesem Hintergrund derzeit von der schulischen Führungsforschung diskutiert werden, sind insbesondere system leadership, distributed leadership, instructional leadership oder aber transformational leadership (Tab. 1).

Bei diesen Führungsstilen handelt es sich aber ,größtenteils um normativ geprägte Beschreibungen, die bestimmte Führungsstile oder -modelle charakterisieren" (Gerick 2014, S. 31) und damit eher Vorstufen einer Führungstheorie darstellen (vgl. Bonsen 2003). Zudem ist darauf hinzuweisen, dass es sich bei schulischem Führungshandeln um ein multidimensionales Konstrukt handelt, das eine hohe Situations- und Kontextbezogenheit aufweist. Bislang existieren also nur wenige integrierende Gesamtdarstellungen von Kontextbedingungen, denen das Führungshandeln unterliegt (vgl. Thillmann et al. 2015; Brauckmann 2012, S. 232). „Den Systemkontext von der Schule, und die Organisation Schule von den Führungshandelnden empirisch zu trennen bzw. die damit angesprochenen Wechselbeziehungen empirisch adäquat zu modellieren, bleibt vorerst ein zentrales Forschungsdesiderat" (vgl. Thillmann et al. 2015, S. 196). Vor dem Hintergrund governance-, organisations- wie auch professionstheoretischer Überlegungen wird gegenwärtig die Idee eines Schnittstellenmanagements stärker konturiert, an der sich ggf. die Effektivität und Effizienz der Handlungskoordination über verschiedene Führungsebenen hinweg zeigt. Dabei geht es vor allem um jene Schnittstellen, an denen ein schulformübergreifender Organi- 
sations- und Führungsprozess von unterschiedlichen Leitungsgremien gemeinsam angegangen werden kann bzw. angegangen werden sollte.

\section{Befunde zum Führungshandeln im Kontext Neuer Steuerungskonzepte}

Insgesamt ist auffällig, dass den führungsbezogenen Anforderungsprofilen der Neuen Steuerung nur wenige empirische Befunde gegenüberstehen. Die Befunde wissenschaftlicher Begleitstudien zum Umgang mit erweiterter schulischer Führungsverantwortung innerhalb und außerhalb der Schule haben deutlich gemacht, dass die im Rahmen der neuen Steuerungskonzepte an SchulleiterInnen gestellten Anforderungen bzw. Erwartungshaltungen unter den derzeit bestehenden Regelungen und Arbeitsbedingungen von SchulleiterInnen nicht immer hinreichend realisiert werden können (Schmerbauch 2017; Huber et al. 2013; Warwas 2012; Languth 2006). Empirische Befunde aus der SHaRP-(Schulleitungshandeln zwischen erweiterten Rechten und Pflichten) Studie weisen darauf hin, ,dass sich in den Tätigkeitsfeldern Organisationsführung und -entwicklung, Personalführung und -entwicklung sowie unterrichtsbezogene Führungsarbeit der zeitliche Aufwand und das Beanspruchungsempfinden von Schulleiterinnen und Schulleitern voneinander unterscheiden. [...] Zugleich sind es gerade diese drei entwicklungsbezogenen Tätigkeitsfelder, denen die Schulleiterinnen und Schulleiter aus SHaRP die höchste Handlungspriorität einräumen“ (Brauckmann et al. 2014, S. 60). Überdies ist es die Wahrnehmung einzelschulischer Problemlagen, seien dies strukturelle Defizite (z.B. bedarfsgerechte Unterrichtsversorgung) oder aber soziale Komponenten (z.B. mangelnde Lernförderung durch die Eltern), welche die Ausübung entwicklungsbezogenen Schulleitungshandelns beschränken kann (Feldhoff und Brauckmann 2013).

Diese Befunde in ihrer Gesamtheit machen deutlich, dass man noch stärker als bisher nach den system-, organisations- und personenbezogenen Bedingungen für die Ausübung, vor allem aber für die Nichtausübung jener führungswie entwicklungsbezogenen Tätigkeiten fragen muss, denen im Rahmen ,Neuer Steuerungsansätze“ eine besondere Bedeutung bei der Sicherung und Steigerung einzelschulischer Qualität zugeschrieben wird. So zielt die Ausübung dieser Tätigkeiten darauf ab, sowohl in der Organisation Schule insgesamt als auch bei den einzelnen Mitarbeiter*innen bislang ungenutzte Ressourcen, Fähigkeiten, Begabungen und Handlungsmöglichkeiten zu erkennen, aufzuspüren und dabei gleichzeitig Freiräume, Anreize und günstige Entwicklungsbedingungen zu schaffen (Brauckmann et al. 2014, S. 60).

Obschon eine weitere Professionalisierung in der Umsetzung der Leitungsfunktion, unter anderem durch die Schulleitungsausbildung und Weiterbildungsangebote, aber auch weitere Entlastungen, insbesondere von Unterrichtsverpflichtungen, im neuen Steuerungsansatz angelegt sind, lässt die derzeitige Befundlage einen wachsenden Unterstützungsbedarf erkennen (Schwanenberg et al. 2018; Schwarz und Brauckmann 2015; Windlinger et al. 2014). Da die rechtlichen Regelungen zur eigenverantwortlichen Schule stark auf die Verantwortung der Schulleitung zuge- 
schnitten sind, wird häufig auf das bislang nicht gelöste Problem hingewiesen, dass das nichtleitende schulische Personal sich nicht hinreichend verantwortlich fühlt oder aber nicht in die Schulentwicklung einbezogen wird (vgl. Hanßen 2013). Diese Tendenz kann sich in dem Maße verringern, wie beispielsweise der Aufbau von Kooperations- und Teamstrukturen gelingt und damit etwa Aktivitäten der schulleiterzentrierten Personalentwicklung ersetzt werden (vgl. Keller-Schneider et al. 2013; Thillmann 2012; Harazd et al. 2011; Feldhoff und Rolff 2008).

\section{Ziel und Anlage des Schwerpunkthefts}

Gegenwärtig ist nicht absehbar, inwieweit sich die Balance zwischen der Stärkung schulischer Eigenständigkeit und der qualitätsbezogenen Rechenschaftspflicht (einschließlich jeweils entsprechender Unterstützungssysteme) in der Praxis als tragfähig erweist. „Es verstärkt sich vielmehr der Eindruck, dass Rechenschaftspflichten eher in Zusammenhang mit dem Ziel schulischer Qualitätssicherung und -entwicklung gesehen werden, als dies bei den „,neuen“ erweiterten Handlungsspielräumen der Fall zu sein scheint" (vgl. Brauckmann 2016, S. 251). Daher ist ein weiterer Klärungsbedarf notwendig, wenn es um die Gewichtung der Rechte und Pflichten von Schulleiterinnen und Schulleitern in unterschiedlichen Schulkontexten geht. Fraglich bleibt somit, ob es sich bei der Einführung Neuer Steuerungskonzepte um eher explizit oder implizit herangetragene Schulleitungsaufgaben handelt, die sich aus Sicht der SchulleiterInnen möglicherweise als unstrukturiert darstellen, da „,ie wenig eindeutig und abgrenzbar, unklar in der Zielsetzung und Lösung sowie in ihren Ursache-Wirkungszusammenhängen kaum überschaubar" sind und zudem nach wie vor ,schwer erkennbar ist, welche Informationen zur Problemlösung notwendig sind und wie diese Informationen beschafft werden können“, wie Döring aus einem anderen Reformanlass bereits 1978 vermutet hatte (Döring 1978, S. 15). So kann eine SchulleiterIn durchaus in Rollenkonflikte geraten, die aus unterschiedlichen Erwartungshaltungen resultieren, wobei das insbesondere für das Spannungsverhältnis zwischen Verwaltung und Pädagogik gilt (vgl. Dröge et al. 1992, S. 8; Krüger 1989, S. 96).

Daher wird in diesem Schwerpunktheft der Frage nachgegangen, inwieweit ein an Qualitätskontrolle (Bildungsstandards, Vergleichsarbeiten, Leistungsvergleichen und Ergebnissen zentraler Prüfungen, Berichte der Schulinspektion) orientiertes Führungshandeln ein autonomes Führungshandeln im Sinne der Qualitätsentwicklung überlagert oder ob sich beide Regelungsmodi tatsächlich hinreichend ergänzen können. So soll nachvollzogen werden, wie mit der Erhöhung der schulischen Eigenverantwortlichkeit neu zugeschriebene Funktionen und Handlungsfelder der Schulleitung verstärkt in das Blickfeld der empirischen Bildungsforschung gerückt sind. Dies betrifft insbesondere aktuelle Probleme und Herausforderungen, die von schulischer Seite mit dem Aufkommen „Neuer“ bildungspolitischer Steuerungsfragen und Neuer Steuerungsmaßnahmen verbunden sind. Damit soll dem komplexen Verhältnis von Steuerungsrealität und Steuerungstheorie Rechnung getragen werden. Für das beobachtbare Schulleitungshandeln zwischen erweiterten Rechten und Pflichten 
bedeutet dies, dass Handlungsoptionen in Abhängigkeit vom jeweiligen schulischen Kontext unterschiedlich bewertet werden (können).

Mit den im Schwerpunktheft vertretenen Beiträgen erfolgt eine Systematisierung der unterschiedlichen Forschungszugänge zum Schulleitungshandeln zwischen erweiterten Rechten und Pflichten. Dabei wird zwischen systemischen, organisationalen und individuellen Einflussgrößen von Schulleitungshandeln (Aufgaben, Rollen, Funktionen, Tätigkeiten, Führungsstilen) differenziert. Dieses Vorgehen ermöglicht differenzierte Betrachtungen von Beziehungs- und Zusammenhangsstrukturen zwischen Neuen Steuerungsansätzen einerseits und Schulleitungshandeln andererseits. Eingeschlossen sind hierbei Wechselbeziehungen zwischen den zwei Strängen neuer Steuerungskonzepte. Aus dieser Konzeptualisierung werden vier übergeordnete Forschungsfragestellungen abgeleitet, die es erlauben, die fachwissenschaftlichen Beiträge im vorliegenden Schwerpunktheft zu gruppieren. Diese lauten im Einzelnen:

1. In welcher Weise sollen Ansätze Neuer Steuerung schulisches Führungshandeln beeinflussen (Funktionserwartungen)?

Altrichter, Kemethofer \& George: „Schulleitungen und evidenzbasierte Bildungsreform im Schulwesen“"

2. Welche Formen von schulischem Führungshandeln sind beobachtbar (Funktionsausübungen)?

Warwas, Helm \& Schadt: Unterstützendes Führungsverhalten schulischer Leitungskräfte für die Arbeit professioneller Lerngemeinschaften im Kollegium

3. Unter welchen Kontextbedingungen entfalten sich bestimmte Formen von Führungshandeln vor Ort (Kontext)?

Pietsch \& Leist: The Effects of Competition in Schooling Markets on Leadership for Learning

Klein \& Bremm: It's almost as if I treat the teachers as I want them to treat the students. Caring als Element von Führung an Schulen in sozial deprivierter Lage (AP)

4. Welche Formen von Führungshandeln prägen die Sicherung und Entwicklung schulischer Qualität (Wirksamkeitsbedingungen)?

Schratz et al.: Schulleitungshandeln an ausgezeichneten Schulen - Erste Einblicke und empirische Befunde

Funding Open access funding provided by University of Klagenfurt.

Open Access Dieser Artikel wird unter der Creative Commons Namensnennung 4.0 International Lizenz (http://creativecommons.org/licenses/by/4.0/deed.de) veröffentlicht, welche die Nutzung, Vervielfältigung, Bearbeitung, Verbreitung und Wiedergabe in jeglichem Medium und Format erlaubt, sofern Sie den/die ursprünglichen Autor(en) und die Quelle ordnungsgemäß nennen, einen Link zur Creative Commons Lizenz beifügen und angeben, ob Änderungen vorgenommen wurden.

\section{Literatur}

van Ackeren, I., \& Bellenberg, G. (2004). Parallelarbeiten, Vergleichsarbeiten und Zentrale Abschlussprüfungen. Bestandsaufnahme und Perspektiven. In H. G. Holtappels, K. Klemm, H. Pfeiffer, H.-G. Rolff \& R. Schulz-Zander (Hrsg.), Jahrbuch der Schulentwicklung Bd. 13. Weinheim: Juventa. 
van Ackeren, I., \& Klemm, K. (2009). Entstehung, Struktur und Steuerung des deutschen Schulsystems. Eine Einführung. Wiesbaden: VS.

Altrichter, H., \& Kemethofer, D. (2015). Neue Ansätze der Steuerung des Schulsystems und die Einstellung von Schulleitungen. Bildung und Erziehung, 68(3), 291-310. https://doi.org/10.7788/bue-20150304.

Altrichter, H., Brauckmann, S., Lassnigg, L., Moosbrugger, R., \& Gartmann, G. B. (2016). Schulautonomie oder die Verteilung von Entscheidungsrechten und Verantwortung im Schulsystem. In M. Bruneforth, F. Eder, K. Krainer, C. Schreiner, A. Seel \& C. Spiel (Hrsg.), Fokussierte Analysen bildungspolitischer Schwerpunktthemen. Nationaler Bildungsbericht Österreich 2015, (Bd. 2, S. 263-304). Graz: Leykam.

Avenarius, H., \& Füssel, H.-P. (2008). Schulrecht im Überblick (2. Aufl.). Darmstadt: Wiss. Buchges.

Bass, B. M. (1985). Leadership and performance beyond expectation. New York: Free Press.

Bellmann, J. (2016). "A tide that lifts all boats"? Neue Steuerung im Schulsystem und die Nachfrage nach Exzellenz. In M. Bonsen \& B. Priebe (Hrsg.), PISA - Folgen und Fragen (S. 105-122). Seelze: Klett.

Bonsen, M. (2003). Schule, Führung, Organisation. Eine empirische Studie zum Organisations- und Führungsverständnis von Schulleiterinnen und Schulleitern. Münster, New York: Waxmann.

Bonsen, M. (2016). Schulleitung und Führung in der Schule. In H. Altrichter \& K. Maag Merki (Hrsg.), Handbuch Neue Steuerung im Schulsystem 2. Aufl. Educational Governance, (Bd. 7, S. 125-148). Wiesbaden: Springer VS.

Böttcher, W. (2017). Schulqualität und der Einfluss der Ökonomie. Skizzen und Thesen zu einem angespannten Verhältnis. In U. Steffens, H. Fend \& K. Maag Merki (Hrsg.), Schulgestaltung. Aktuelle Befunde und Perspektiven der Schulqualitäts- und Schulentwicklungsforschung: Grundlagen der Qualität von Schule. Beiträge zur Schulentwicklung, (Bd. 2, S. 119-184). Münster: Waxmann.

Böttcher, W., Bos, W., Döbert, H., \& Holtappels, H. G. (Hrsg.). (2008). Bildungsmonitoring und Bildungscontrolling in nationaler und internationaler Perspektive. Dokumentation zur Herbsttagung der Kommission Bildungsorganisation, -planung, -recht $(K B B B)$. Münster: Waxmann.

Brauckmann, S. (2012). Schulleitungshandeln zwischen deconcentration, devolution und delegation (3D). empirische Annäherungen aus internationaler Perspektive. Empirische Pädagogik, 26(1), 76-100.

Brauckmann, S. (2016). Schulleitungshandeln zwischen erweiterten Rechten und Pflichten (SHaRP) unter Berücksichtigung einschlägiger schulrechtlicher Bestimmungen. In BMBF (Hrsg.), Steuerung im Bildungssystem (S. 238-255). Bielefeld: wbv.

Brauckmann, S., \& Schwarz, A. (2015). No time to manage? The trade-off between relevant tasks and actual priorities of school leaders in Germany. International Journal of Educational Management, 29(6), 749-765.

Brauckmann, S., Herrmann, C., Hanßen, K. D., Böse, S., Holz, T., \& Feldhoff, T. (2014). Ergebnisbericht im Rahmen des BMBF Forschungsschwerpunkts ,Steuerung im Bildungssystem “ (SteBis) geförderten Forschungsprojekts „Schulleitungshandeln zwischen erweiterten Rechten und Pflichten (SHaRP)“. Frankfurt am Main, Berlin. https://www.dipf.de/de/forschung/aktuelle-projekte/pdf/steubis/projektsharp-pdf/a_Brauckmann_AbschlusstagungKurzvortrag.pdf.

Brauckmann, S., Kühne, S., Stäsche, U., Tarazona, M., Weishaupt, H., \& Wittmann, E. (2010). Steuerung und Verwaltung des Bildungswesens. In R. S. Jäger, P. Nenniger, H. Petillon, B. Schwarz \& B. Wolf (Hrsg.), Grundlegende empirische pädagogische Forschung. Empirische Pädagogik 1990-2010, (Bd. 1, S. 119-160). Landau: Verlag Empirische Pädagogik.

Burns, J. M. (1978). Leadership. New York: Harper \& Row.

De Bevoise, W. (1984). Synthesis of research on the principal as instructional leader. Educational Journal, 41(5), 14-20.

Döring, P. A. (Hrsg.). (1978). Führungsaufgaben der Schulleitung. Schulleiter-Handbuch, Bd. 3. Braunschweig: Westermann.

Dröge, J., Pfeffer, P., \& Thies, H.-H. (1992). Aufgabenfelder der Schulleitung. Schulleiter-Handbuch, Bd. 61. Braunschweig: VS.

Feldhoff, T., \& Rolff, H.-G. (2008). Einfluss von Schulleitungs- und Steuergruppenhandeln. In H. G. Holtappels, K. Klemm, H.-G. Rolff \& H. Pfeiffer (Hrsg.), Schulentwicklung durch Gestaltungsautonomie. Ergebnisse der Begleitforschung zum Modellvorhaben „selbstständige Schule“ in NordrheinWestfalen (S. 293-303). Münster: Waxmann.

Feldhoff, T., \& Brauckmann, S. (2013, April). May the structure be with you! Uncovering the magic triangle of organisational structure, perception and newly assigned leadership activities in Germany. Empirical insights from the School Leaders' Activities. Incentivizing Productive Leadership Practices: A Cross-national Comparison of School Leadership under Alternative Governance and Accountability between More Responsibility and More Power (SHaRP)-Study. San Francisco. 
Gerick, J. (2014). Transformationale Führung aus Lehrersicht und ihre Bedeutung für die Lehrergesundheit. In K. Drossel, R. Strietholt \& W. Bos (Hrsg.), Empirische Bildungsforschung und evidenzbasierte Reformen im Bildungswesen (S. 109-127). Münster: Waxmann.

de Grauwe, A. (2004). School-based management (SBM). Does it improve quality? Paper commissioned for the EFA Global Monitoring Report (UNESCO).

Gronn, P. (2000). Distributed properties: a new architecture for leadership. Educational Management Administration \& Leadership, 28, 317-338.

Hanßen, K. (2013). Rechtliche Regelungen zu Tätigkeitsfeldern von Schulleiterinnen und Schulleitern bei erweiterter Eigenverantwortung von Schule. Eine Untersuchung der Rechtslage in den Ländern Berlin und Niedersachsen (Ergänzung zu der Untersuchung der Rechtslage in den Ländern Bayern, Hessen und Nordrhein-Westfalen sowie in den Ländern Brandenburg und Hamburg). https://www.dipf. de/de/forschung/aktuelle-projekte/pdf/steubis/projekt-sharp-pdf/Endfassung_BE_NI.pdf.

Harazd, B., Gieske, M., \& Gerick, J. (2011). Schulleitung in eigenverantwortlichen Schulen. In F. Dietrich (Hrsg.), Neue Steuerung - alte Ungleichheiten? Steuerung und Entwicklung im Bildungssystem (S. 101-112). Münster: Waxmann.

Hattie, J. (2009). Visible learning. A synthesis of over 800 meta-analyses relating to achievement. London: Routledge.

Heinrich, M., \& Kohlstock, B. (Hrsg.). (2016). Ambivalenzen des Ökonomischen. Analysen zur „Neuen Steuerung “ im Bildungssystem (1. Aufl.). Educational Governance, Bd. 29. Wiesbaden: Springer VS. https://doi.org/10.1007/978-3-658-10084-1.

Heinrich, M., \& Kussau, J. (2016). Das Schulprogramm zwischen schulischer Selbstregelung und externer Steuerung. In H. Altrichter \& K. Maag Merki (Hrsg.), Handbuch Neue Steuerung im Schulsystem 2. Aufl. Educational Governance, (Bd. 7, S. 183-208). Wiesbaden: Springer VS.

Herrmann, U.G. (2009). „Alte“ und „,neue“ Steuerung im Bildungssystem. Anmerkungen zu einem bildungshistorisch problematischen Dualismus. In U. Lange, S. Rahn, W. Seitter \& R. Körzel (Hrsg.), Steuerungsprobleme im Bildungswesen (VS research Theorie und Empirie lebenslanges Lernen (TELLL) (S. 57-77). Wiesbaden: VS.

Hopkins, D. (2008). Realising the potential of system leadership. In B. Pont, D. Nusche \& D. Hopkins (Hrsg.), Case Studies on System Leadership (Bd. 2, S. 21-36). Paris: OECD.

Huber, S. G. (2010). System Leadership - systemische Schulentwicklung durch Kooperation. Journal für Schulentwicklung, 14(2), 8-21.

Huber, S.G., Wolfgramm, C., \& Kilic, S. (2013). Vorlieben und Belastungen im Schulleitungshandeln: Ausgewählte Ergebnisse aus der Schulleitungsstudie 2011/2012 in Deutschland, Österreich, Liechtenstein und der Schweiz. In S. G. Huber (Hrsg.), Schwerpunkt. Gesunde Schule. Jahrbuch Schulleitung, (Bd. 2013, S. 259-271). Köln: Link.

Kanape, A., \& Kemethofer, D. (2017). Schulleitung in Österreich: Zwischen Steuerung und Autonomie. In A. Paseka, M. Heinrich, A. Kanape \& R. Langer (Hrsg.), Schulentwicklung zwischen Steuerung und Autonomie. Beiträge aus Aktions-, Schulentwicklungs- und Governance-Forschung (S. 119-138). Münster: Waxmann.

Keller-Schneider, M., Albisser, S., \& Wissinger, J. (Hrsg.). (2013). Professionalität und Kooperation in Schulen. Beiträge zur Diskussion über Schulqualität. Bad Heilbrunn: Klinkhardt.

Klein, E. D. (2017). Autonomy and accountability in schools serving disadvantaged communities. Journal of Educational Administration, 55(5), 589-604. https://doi.org/10.1108/JEA-06-2016-0065.

Krüger, R. (1989). Schulleiteralltag. Beobachtungen und Analysen. Schulleiter-Handbuch, Bd. 51. Braunschweig: SL.

Languth, M. (2006). Schulleiterinnen und Schulleiter im Spannungsverhältnis zwischen programmatischen Zielvorgaben und alltäglicher Praxis. Eine empirische Studie zur Berufsauffassung von Schulleiterinnen und Schulleitern. Dissertation, Göttingen.

Pietsch, M., \& Tulowitzki, P. (2017). Disentangling school leadership and its ties to instructional practices - an empirical comparison of various leadership styles. School Effectiveness and School Improvement, 2(4), 629-649. https://doi.org/10.1080/09243453.2017.1363787.

Pont, B., Nusche, D., \& Moorman, H. (2008). Policy and practice. Improving school leadership, Bd. 1. Paris: OECD.

Rolff, H.-G. (2009). Führung als Gestaltung und ihre Bedeutung für die Schulreform. Die Deutsche Schule, 101(3), 253-265.

Schley, W., \& Schratz, M. (2010). Developing leaders, building networks, changing schools through system leadership. In J. MacBeath \& T. Townsend (Hrsg.), International handbook on leadership for learning (S. 267-296). New York: Springer.

Schmerbauch, A. (2017). Schulleitung und Schulsteuerung. Wiesbaden: Springer. 
Schratz, M., Wiesner, C., Kemethofer, D., George, A.C., Rausch, A., Krenn, S., \& Huber, S. G. (2016). Schulleitung im Wandel: Anforderungen an eine ergebnisorientierte Führungskultur. In M. Bruneforth, F. Eder, K. Krainer, C. Schreiner, A. Seel \& C. Spiel (Hrsg.), Fokussierte Analysen bildungspolitischer Schwerpunktthemen. Nationaler Bildungsbericht Österreich, (Bd. 2, S. 221-262). Graz: Leykam.

Schümer, G., \& Weiss, M. (2008). Bildungsökonomie und Qualität der Schulbildung. Kommentar zur bildungsökonomischen Auswertung von Daten aus internationalen Schulleistungsstudien. Frankfurt: GEW.

Schwanenberg, J., Klein, E.D., \& Walpuski, M. (Hrsg.). (2018). Wie erfolgreich fühlen sich Schulleitungen und welche Unterstützungsbedürfnisse haben sie? Ergebnisse aus dem Projekt Schulleitungsmonitor. SHIP Working Paper Reihe. Essen. https://duepublico.uni-duisburg-essen.de/servlets/ DerivateServlet/Derivate-46364/Schwanenberg_et_al_Schulleitungen_SHIP_3.pdf.

Smith, W.F., \& Andrews, R.L. (1989). Instructional leadership: how principals make a difference. Alexandria: ASCD.

Specht, W. (2008). Innovation durch Evaluation. In Landesinstitut für deutschsprachige Länder in Potsdam, Bundesministerium für Unterricht, Kunst und Kultur \& Schweizerische Konferenz der kantonalen Erziehungsdirektion (Hrsg.), Bildungsmonitoring, Vergleichsstudien und Innovationen. Von evidenzbasierter Steuerung zur Praxis. OECD/CERI-Regionalseminar für die deutschsprachigen Länder, Potsdam, 25.28.2007. (S. 41-52). Berlin: Berliner Wiss.-Verl..

Thillmann, K. (2012). Schulentwicklung und Schulorganisation. Eine empirische Untersuchung schulischer Organisationsgestaltung vor dem Hintergrund der Neuen Steuerung im Bildungssystem. Freie Universität Berlin: Dissertation.

Thillmann, K., Brauckmann, S., Hermann, C., \& Thiel, F. (2015). Praxis schulischer Personalentwicklung unter den Bedingungen der Neuen Steuerung. Empirische Befunde aus Forschungsprojekten SHaRP und StABil. In H. J. Abs, T. Brüsemeister, M. Schemmann \& J. Wissinger (Hrsg.), Governance im Bildungssystem. Analysen zur Mehrebenenperspektive, Steuerung und Koordination. Educational Governance, (Bd. 26, S. 195-228). Wiesbaden: Springer VS.

Warwas, J. (2012). Berufliches Selbstverständnis, Beanspruchung und Bewältigung in der Schulleitung. Wiesbaden: VS.

Windlinger, R., Hostettler, U., \& Kirchhofer, R. (2014). Schulleitungshandeln, Schulkontext und Schulqualität. Eine quantitative Untersuchung der komplexen Beziehungen am Beispiel des deutschsprachigen Teils des Kantons Bern. Bern. Forschungsprojekt. http://schulleitungshandeln.ch/files/2012/ 04/Schlussbericht_DORE_140305_Layout_definitiv.pdf.

Wissinger, J. (2016). Schulleitung im Fokus des Schulqualitätsdiskurses. In U. Steffens \& T. Bargel (Hrsg.), Schulqualität - Bilanz und Perspektiven. Grundlagen der Qualität von Schule 11 . Aufl. Beiträge zur Schulentwicklung. (S. 257-276). Münster: Waxmann. 